\title{
Consistency Analysis in Multi-language Knowledge Sharing System
}

- Authors

- Authors and affiliations

- Amit Pariyar

- Yohei Murakami

- Donghui Lin

- Toru Ishida

Chapter

First Online: 24 February 2018

- 336Downloads

Part of the Cognitive Technologies book series (COGTECH)

\section{Abstract}

Unprecedented growth in knowledge sharing among multi-language communities, both common and distinct languages, has raised the possibility of sharing inconsistent content. Though popular with traditional system, the approach to explicitly state consistency rules to avoid inconsistency is practically not suited for multi-language knowledge sharing system because of sheer complexity. Alternatively this chapter focuses on potential cause of inconsistency, cases such as content omitted, content updates not propagated and content conflicts. Ignoring such cases in knowledge sharing has undesirable consequences: community bias, global and local inconsistency and regional discrepancies. Consistency constraints from opposing knowledge sharing goals among communities is another issue. Due to which consistency policy ranges from rigid 'one to one consistency' to non-rigid 'consistency where needed'. This chapter contributes with (a) process-based approach for multilingual content synchronization to leverage knowledge equally and (b) propagation-based approach to analyze community preferences when sharing specific content categories/geographic regions, to customize knowledge sharing; a value add-on to designing language services adhering to knowledge sharing goals.

\section{Keywords}

Inconsistency Multi-language Knowledge sharing Content category Geographic region 


\section{Copyright information}

(C) Springer Nature Singapore Pte Ltd. 2018

About this chapter

CrossMark

\section{Cite this chapter as:}

Pariyar A., Murakami Y., Lin D., Ishida T. (2018) Consistency Analysis in Multi-language Knowledge Sharing System. In: Murakami Y., Lin D., Ishida T. (eds) Services Computing for Language Resources. Cognitive Technologies. Springer, Singapore.

https://doi.org/10.1007/978-981-10-7793-7_9

- $\quad$ First Online24 February 2018

- DOIhttps://doi.org/10.1007/978-981-10-7793-7_9

- Publisher NameSpringer, Singapore

- $\quad$ Print ISBN978-981-10-7792-0

- Online ISBN978-981-10-7793-7

- eBook PackagesComputer ScienceComputer Science (R0) 